BULLETIN OF THE

AMERICAN MATHEMATICAL SOCIETY

Volume 80, Number 5, September 1974

\title{
SOME THEOREMS ON $C$-FUNCTIONS
}

\author{
BY LESLIE COHN $^{1}$
}

Communicated by E. M. Stein, November 29, 1973

The purpose of this note is to announce certain results $I$ have obtained about the behavior of the Harish-Chandra $C$-function as a meromorphic function. The notation and terminology, if not explained, are that of [2], [3], or [6].

1. The $C$-ring. Let $(P, A)$ be a fixed parabolic pair of a semisimple Lie group $G$ having finite center, $P=M A N$ the corresponding Langlands decomposition, $K$ a fixed maximal compact subgroup. Let $\mathscr{G}, \mathscr{K}, \mathscr{K}_{M}$, $\mathscr{M}$ be the universal enveloping algebras of $G, K, K_{M}$, and $M$, respectively $\left(K_{M}=K \cap M\right)$-i.e. of their complexified Lie algebras $\mathfrak{g}_{C}, \mathfrak{f}_{C}, \mathfrak{f}_{M, C}, \mathrm{~m}_{C}$. Let $b \rightarrow b^{\iota}(b \in \mathscr{G})$ denote the unique anti-automorphism of $\mathscr{G}$ such that $X^{\iota}=-X(X \in \mathfrak{g})$. Consider $\mathscr{K}$ to be a right $\mathscr{K}_{M}$-module via the multiplication in $\mathscr{K}: b \circ d=b d\left(b \in \mathscr{K}, d \in \mathscr{K}_{M}\right)$, and consider $\mathscr{M}$ to be a left $\mathscr{K}_{M}$-module via the operation $d \circ c=c d^{\imath}\left(d \in \mathscr{K}_{M}, c \in \mathscr{M}\right)$. We can then form the tensor product $\mathscr{K} \otimes \mathscr{K}_{M} \mathscr{M}$ of $\mathscr{K}_{M}$-modules. (We write $b \hat{\otimes} c$ for elements of $\mathscr{K} \otimes \mathscr{K}_{M} \mathscr{M}, b \otimes c$ for elements of $\mathscr{K} \otimes \mathscr{M}$.) The group $K_{M}$ acts on $\mathscr{K} \otimes \mathscr{K}_{M} \mathscr{M}$ via the (well-defined) representation $\rho: \rho(m)(b \hat{\otimes} c)=b^{m} \hat{\otimes} c^{m}\left(b \in \mathscr{K}, c \in \mathscr{M}, m \in K_{M}\right)$. Let $\left(\mathscr{K} \otimes \mathscr{K}_{M} \mathscr{M}\right)^{K_{M}}$ denote the $K_{M}$-invariants.

Proposition 1. $\left(\mathscr{K} \otimes \mathscr{K}_{M} \mathscr{M}\right)^{K_{M}}$ is a ring (i.e., the "obvious" multiplication is well defined). In fact, it is a left and right Noetherian integral domain (noncommutative, in general), hence has a quotient division algebra.

We refer to $\left(\mathscr{K} \otimes \mathscr{K}_{, M} \mathscr{M}\right)^{K_{M}}$ as the $C$-ring associated to the pair $(P, A)$.

Let $\tau$ be a left or double representation of $K$ on a finite-dimensional Hilbert space $V$. Then there exists a representation $\lambda_{\tau}$ of the ring $\mathscr{K} \otimes \mathscr{M}$ on $C^{\infty}(M: V)$ defined as follows:

$\lambda_{\tau}(b \otimes c) \psi(m)=\tau(b) \psi\left(c_{i}^{l} m\right) \quad\left(b \in \mathscr{K}, c \in \mathscr{M}, m \in M, \psi \in C^{\infty}(M: V)\right)$.

Let $C^{\infty}\left(M, \tau_{M}\right)$ denote the space of $\psi \in C^{\infty}(M: V)$ such that

$$
\tau(k) \psi(m)=\psi(k m) \quad\left(k \in K_{M}, m \in M\right)
$$

if $\tau$ is a left representation of $K$ or such that

$$
\tau\left(k_{1}\right) \psi(m) \tau\left(k_{2}\right)=\psi\left(k_{1} m k_{2}\right) \quad\left(k_{1}, k_{2} \in K_{M}, m \in M\right)
$$

AMS (MOS) subject classifications (1970). Primary 43A90, 33A75; Secondary 39A10.

${ }^{1}$ Research partially supported by NSF Grants GP-25320 and GP37121. 
if $\tau$ is a double representation of $K$ on $V$. Then the rule

$$
\lambda_{r}\left(\sum b_{j} \hat{\otimes} c_{j}\right) \psi(m)=\sum \tau\left(b_{j}\right) \psi\left(c_{j i}^{l} m\right) \quad\left(b_{j} \in \mathscr{K}, c_{j} \in \mathscr{M}\right)
$$

defines a representation of the $C$-ring $\left(\mathscr{K} \otimes \mathscr{K}_{M} \mathscr{M}\right)^{K_{M}}$ on $C^{\infty}\left(M, \tau_{M}\right)$. Clearly the spaces $\mathscr{C}\left(M, \tau_{M}\right)$ and ${ }^{\circ} \mathscr{C}\left(M, \tau_{M}\right)$ of Schwartz functions and cusp forms in $C^{\infty}\left(M, \tau_{M}\right)$ respectively are invariant subspaces.

2. The difference equations satisfied by the $C$-function. By a polynomial function on a connected simply connected nilpotent Lie group $N$, we mean a function $f \in C^{\infty}(N)$ such that $X \rightarrow f(\exp X)(X \in L(N))$ is a polynomial function on the Lie algebra $L(N)$ of $N$.

By a semilattice $L$ in a real vector space $V$, we mean an additive semigroup generated by a basis of $V$.

Proposition 2. There exists a semilattice $L \subseteq \mathfrak{a}^{*}$ such that $\mu \in L$ implies that $e^{2 \mu(H(\bar{n}))}$ is a polynomial function on $\bar{N}$.

THEOREM 1 (The difference equations). Let $\mu \in \mathfrak{a}^{*}$ be such that $e^{2 \mu(H(\bar{n}))}$ is a polynomial function on $\bar{N}$. Then there exist polynomials $b^{\mu}(v), c^{\mu}(v)$ with coefficients in the C-ring $\left(\mathscr{K} \otimes \mathscr{K}_{M} \mathscr{M}\right)^{K_{M}}$ such that, for all double unitary representations $\tau$ of $K$,

$$
\lambda_{\tau}\left(b^{\mu}(v)\right) C_{P \mid P}(1: v)=\lambda_{\tau}\left(c^{\mu}(v)\right) C_{P \mid P}(1: v-2 i \mu) \quad\left(\nu \in \mathfrak{a}_{C}^{*}\right) .
$$

The polynomials $b^{\mu}(v)$ and $c^{\mu}(v)$ have the same degree and the same leading term, which we may assume lies in $C[v]$ (i.e., is a scalar polynomial). The coefficients of $c^{\mu}(v)$, in fact, lie in the subring $3_{M}$ (the center of $\mathscr{M})$ of the $C$-ring. The operators $\lambda_{\tau}\left(b^{\mu}(v)\right), \lambda_{\tau}\left(c^{\mu}(v)\right)$ are never identically zero (as polynomials in $\nu$ ).

Taking $\mu=\mu_{1}, \cdots, \mu_{l}$ to be generators of a semilattice $L$ as in Proposition 2 , we get the result that the $C$-function $C_{P \mid P}(1: v)$ satisfies a system of $l=r k P$ linear first order partial difference equations with polynomial coefficients.

\section{The asymptotic development.}

THEOREM 2. Choose $\lambda \in \mathfrak{a}_{C}^{*}$ such that $\operatorname{Re}\langle\lambda, \alpha\rangle>0$ for all roots $\alpha$ of the pair $(P, A)$. Then there exists a formal power series $\sum_{j=0}^{\infty} t^{-j} b_{j}^{(\lambda)}(v)$ with

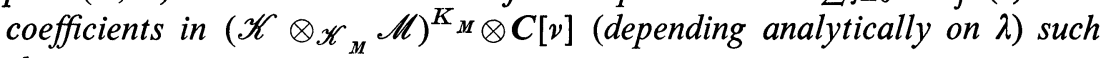
that

(1) $b_{0}^{(\lambda)}(v) \in C$;

(2) $b_{j}^{(\lambda)}(v)$ is of degree at most $2 j$ in $\nu(j \geqq 0)$; and

(3) for every double representation $\tau$ of $K$,

$$
C_{P \mid P}(1: \nu+i t \lambda) \sim t^{-s / 2} \sum_{j=0}^{\infty} t^{-j} \lambda_{\tau}\left(b_{j}^{(\lambda)}(\nu)\right) \quad \text { as } t \rightarrow \infty
$$


uniformly for $v$ in compact subsets of $\mathrm{a}_{C}^{*}$ (both sides being considered as operators on the space $\left.{ }^{\circ} \mathscr{C}\left(M, \tau_{M}\right)\right)$. This means that, for each integer $n \geqq 0$,

$$
\lim _{t \rightarrow \infty} t^{n}\left|t^{s / 2} C_{P \mid P}(1: v+i t \lambda)-\sum_{j=0}^{n} t^{-j} \lambda_{\tau}\left(b_{j}^{(\lambda)}(v)\right)\right|=0 .
$$

Here $s=\operatorname{dim} N$. Replacing $\tau$ by the trivial representation of $K$, we get the same asymptotic expansion for the integral $\underline{C}(v)=\int_{\bar{N}} e^{i v-\rho(H(\bar{n}))} d \bar{n}$.

CoROllary 1. Suppose that $\lambda$ is as in Theorem 2. Then there exists a constant $\zeta_{\lambda}$ such that

$$
\lim _{t \rightarrow \infty} t^{s / 2} C_{P \mid P}(1: \nu+i t \lambda)=\zeta_{\lambda} \times \text { id }
$$

as an operator on ${ }^{\circ} \mathscr{C}\left(M, \tau_{M}\right)$, the limit being uniform in $v$ on compact subsets of $a_{C}^{*}$.

\section{The representation theorems.}

THeORem 3. Choose $\mu \in \mathfrak{a}^{*}$ such that $\langle\mu, \alpha\rangle>0$ for all roots $\alpha$ of $(P, A)$ and $e^{2 \mu(H(\bar{n}))}$ is a polynomial function on $\bar{N}$. Let $b(v)=b^{\mu}(v), c(v)=$ $c^{\mu}(v)$ be as in Theorem 1 . Then

$$
\begin{aligned}
C_{P \mid P}(1: v)=\text { const } \times \lim _{n \rightarrow \infty} n^{-s / 2} \lambda_{\tau}(c(v+2 i \mu) & \cdots c(v+2 i n \mu))^{-1} \\
& \times \lambda_{\tau}(b(v+2 i \mu) \cdots b(v+2 i n \mu))
\end{aligned}
$$

(the constant being independent of $\tau$ ).

THEOREM 4. Let $(P, A)$ be an arbitrary parabolic subgroup of $G$; and let $\tau$ be a double unitary representation of $K$. Then there exist $\mu_{1}, \cdots, \mu_{r} \in \mathfrak{a}^{*}$ and constants $p_{i j}, q_{i j}\left(i=1, \cdots, r, j=1, \cdots, j_{i}\right)$ depending on $\tau$ such that

$$
\operatorname{det} C_{P \mid P}(1: \nu)=\mathrm{const} \times \prod_{i=1}^{r} \prod_{j=1}^{j_{i}} \frac{\Gamma\left(-i\left\langle\nu, \alpha_{i}\right\rangle / 2\left\langle\mu_{i}, \alpha_{i}\right\rangle+q_{i j}\right)}{\Gamma\left(-i\left\langle\nu, \alpha_{i}\right\rangle / 2\left\langle\mu_{i}, \alpha_{i}\right\rangle+p_{i j}\right)},
$$

where $\alpha_{1}, \cdots, \alpha_{r}$ are the reduced roots of $(P, A)$.

Open Question. Are the numbers $p_{i j}, q_{i j}$ always rational?

5. Idea of the proofs. Theorem 1 is based on the following sequence of results.

Fix a Cartan subalgebra $\mathfrak{h}$ of $\mathfrak{g}$ containing $\mathfrak{a}$; and let $P_{+}$denote the set of roots $\beta$ of $\left(\mathfrak{g}_{C}, \mathfrak{h}_{C}\right)$ such that $\beta \mid \mathfrak{a}>0$. Let $X_{\beta}, X_{-\beta}\left(\beta \in P_{+}\right)$be root vectors such that $B\left(X_{\beta}, X_{-\beta}\right)=1$ and $\theta\left(X_{\beta}\right)=-\bar{X}_{-\beta}=-X_{\theta \beta}$.

Define vector fields $q(X)(X \in \mathfrak{g})$ on $\bar{N}=\theta(N)$ by the following rule:

$$
q(X) f(\bar{n})=-\sum_{\beta \in P_{+}} B\left(X, X_{\beta}^{\bar{n}}\right) f\left(\bar{n} ; X_{-\beta}\right) \quad\left(f \in C^{\infty}(\bar{N})\right) .
$$


Proposition 3. $\quad X \rightarrow q(X)$ defines a representation of $\mathfrak{g}$ by derivations of $C^{\infty}(\bar{N})$. The ring $\mathscr{R}_{\bar{N}}$ of polynomial functions on $\bar{N}$ is a q-invariant subspace of $C^{\infty}(\bar{N})$.

Let $\sum_{0}(P, A)=\left\{\alpha_{1}, \cdots, \alpha_{l}\right\}$ be the simple roots of $(P, A)$; and choose $H_{j} \in \mathfrak{a}$ such that $\alpha_{i}\left(H_{j}\right)=\delta_{i j}$.

Proposition 4. Suppose that $X \in \mathfrak{g}$. Then

$$
e_{v}\left(X_{i} x\right)=\left\{\sum_{j=1}^{l}\left\langle i v-\rho, \alpha_{j}\right\rangle B\left(X, H_{j}^{k(x)}\right)\right\} e_{\nu}(x) \quad(x \in G) .
$$

( $B$ is the Killing form on $\mathrm{g} ; e_{\nu}(x)=e^{i \nu-\rho(H(x))}$.)

Corollary 2. Suppose that $Z \in \mathfrak{F}$. Then

$$
q(Z) e_{v}(\bar{n})=\left\{\sum_{j=1}^{l}\left\langle i v-\rho, \alpha_{j}\right\rangle B\left(Z, H_{j}^{\bar{n}}\right)\right\} e_{v}(\bar{n}) \quad(\bar{n} \in \bar{N}) .
$$

Let $V_{1}, \cdots, V_{t}(t=\operatorname{dim} \mathrm{m})$ be an orthonormal basis for $m_{C_{A}}$ (with respect to the Killing form). Also, given $\psi \in C^{\infty}\left(M, \tau_{M}\right)$, define $\hat{\Psi}: G \rightarrow$ $C^{\infty}(M: V)$ by

$$
\hat{\Psi}(x \mid m)=\psi(x m) \quad(x \in G, m \in M) .
$$

Proposition 5. Suppose that $Z \in \mathfrak{f}$ and $\psi \in C^{\infty}\left(M, \tau_{M}\right)$. Then $\lambda_{\tau}(Z \otimes 1) \hat{\Psi}(\bar{n})=-q(Z) \hat{\Psi}(\bar{n})-\sum_{j} B\left(Z, V_{j}^{\bar{n}}\right) \lambda_{r}\left(1 \otimes V_{j}\right) \hat{\Psi}(\bar{n}) \quad(\bar{n} \in \bar{N})$

Proposition 6. There exists a unique $\mathscr{M} \otimes C[v]$ module homomorphism

such that

$$
F: \mathscr{K} \otimes \mathscr{K}_{M} \mathscr{M} \otimes C[v] \rightarrow \mathscr{M} \otimes \mathscr{R}-\otimes C[v]
$$

(1) $F(1)=1$;

(2) $F(v \mid \bar{n})(Z)=\sum\left\langle i v+\rho, \alpha_{j}\right\rangle B\left(Z, H_{j}^{\bar{n}}\right)-\sum B\left(Z, V_{j}^{\bar{n}}\right) V_{j} \quad(Z \in \mathfrak{f})$;

(3) $F(Z b)=F(b) F(Z)+q(Z) F(b) \quad(Z \in \mathfrak{F}, b \in \mathscr{K})$;

(4) $F(b \hat{\otimes} c)=c F(b) \quad(b \in \mathscr{K}, c \in \mathscr{M})$.

Proposition 7. Suppose that $b \in \mathscr{K} \otimes \mathscr{K}_{M} \mathscr{M}$. Then there exists a constant $C=C(b)>0$ such that if $\operatorname{Im}\left\langle v, \alpha_{j}\right\rangle \geqq C(b)(j=1, \cdots, l)$, then $\lambda_{\tau}(b) \int_{\bar{N}} e_{v}(\bar{n}) \psi(\bar{n} m) d \bar{n}=\int_{\bar{N}} e_{v}(\bar{n}) \lambda_{\tau}(1 \otimes F(v \mid \bar{n})(b)) \hat{\Psi}(\bar{n} \mid m) d \bar{n} \quad(m \in M)$, both integrals being convergent.

Proposition 8. Given $\phi(\bar{n}) \in \mathscr{R}_{\bar{N}}$, we can find $b(v) \in \mathscr{K} \otimes \mathscr{K}_{M} \mathscr{M} \otimes$ $C[v]$ and $c(v) \in 3_{M} \otimes C[v]$ such that $F(b(v))=c(v) \phi$. 
ProOF OF THEOREM 1. First apply Proposition 8 with $\phi(\bar{n})=e^{2 \mu(H(\tilde{n}))}$. Then apply Proposition 7.

The following is the essential step in the proof of Theorem 2.

Proposition 9. Suppose that $v \in \mathfrak{a}_{C}^{*}$ and let $f_{\nu}(\bar{n})=v(H(\bar{n}))(\bar{n} \in \bar{N})$. Then if $\langle\nu, \alpha\rangle \neq 0$ for all $\alpha \in \sum(P, A), \bar{n}=e$ is the only critical point of $f_{v}$, and $\bar{n}=e$ is a nondegenerate critical point. Furthermore if $\nu \in \mathfrak{a}^{*}$ and $\langle\nu, \alpha\rangle>0$ for all $\alpha \in \sum(P, A)$, then the critical point of the (real-valued) function $f_{\nu}(\bar{n})$ has index 0 .

Proposition 9 allows us to apply the method of steepest descent (see [1]) to derive the asymptotic expansion of $C_{\vec{P} \mid P}(1: v)$ (Theorem 2).

Theorems 3 and 4 follow fairly easily, given Theorems 1 and 2.

6. An example: the $C$-function for the group $S U(1,2)$. In this case, the set $P_{+}$consists of three roots $\beta_{1}, \beta_{2}$ and $\beta_{3}$, where $\beta_{1}$ and $\beta_{2}$ are simple and $\beta_{3}=\beta_{1}+\beta_{2}$. Also, the parabolic pair $(P, A)$ is minimal; so the $C$-ring is isomorphic to $\mathscr{K}^{M}$. If $\mu=\alpha$ (the simple root of $\left.(P, A)\right), e^{2 \mu(H(\bar{n}))}$ is a polynomial function on $\bar{N}$; the corresponding polynomials $b^{\mu}(\nu)$ and $c^{\mu}(v)$ are then as follows

where

$$
b^{\mu}(\nu)=b_{1}^{\mu}(\nu) b_{2}^{\mu}(v)
$$

$$
\begin{aligned}
& b_{1}^{\mu}(v)=\left\{\left(\langle i v+\rho, \alpha\rangle-i(\sqrt{ } 6 / 6) Z_{\beta_{3}}\right)\left(\langle i v, \alpha\rangle+\frac{1}{2} V\right)+\frac{1}{3} Z_{\beta_{1}} Z_{\beta}^{2}\right\} \\
& b_{2}^{\mu}(v)=\left\{\left(\langle i v+\rho, \alpha\rangle-\frac{1}{2} V\right)\left(\langle i v, \alpha\rangle+i(\sqrt{ } 6 / 6) Z_{\beta_{3}}\right)+\frac{1}{3} Z_{\beta_{1}} Z_{\beta_{2}}\right\}
\end{aligned}
$$

and

$$
c^{\mu}(v)=\langle i v+\rho, \alpha\rangle\langle i v+\alpha, \alpha\rangle\left(\langle i v+\rho, \alpha\rangle+\frac{1}{2} V\right)\left(\langle i v+\rho, \alpha\rangle-\frac{1}{2} V\right) .
$$

Here $Z_{\beta_{\imath}}=\frac{1}{2}\left(X_{\beta_{i}}+\theta\left(X_{\beta_{i}}\right)\right)\left(X_{\beta_{\imath}}\right.$ normalized as above), and $V$ is the element of $\mathrm{m}_{C}$ such that $\beta_{1}(V)=\frac{1}{2}$.

Using the polynomials $b^{\mu}(v)$ and $c^{\mu}(v)$, we obtain the following result.

Proposition 10. Let $\tau=\tau_{m, n}$ be the $(m+1)$-dimensional representation of $K=U(2)$ such that $\tau\left(V-i \sqrt{ } 6 Z_{\beta_{3}}\right)=n \times 1$. $\left(V-i \sqrt{ } 6 Z_{\beta_{3}}\right.$ spans the center of $\mathfrak{\mathfrak { F }}_{C}$.) Let $\mathscr{V}=\mathscr{V}^{(m, n)}$ denote the space of $\tau$. Then $\mathscr{V}$ has a basis $x_{j}(j=0,1, \cdots, m)$ such that $\tau(V) x_{j}=\frac{1}{4}(m+n-2 j) x_{j}$. Furthermore, the operator

$$
C_{\bar{P} \mid P}^{(m, n)}(1: v)=\int_{\bar{N}} \tau(k(\bar{n})) e^{i v-\rho(H(\bar{n}))} d \bar{n}
$$

on $\mathscr{V}^{(m, n)}$ has each vector $x_{j}$ as an eigenvector; and the corresponding eigenvalue is

$$
\frac{2}{\sqrt{ } \pi} \frac{\Gamma\left(\zeta_{1}\right) \Gamma\left(\zeta_{2}\right) \Gamma\left(\zeta_{3}\right) \Gamma\left(\zeta_{4}\right)}{\Gamma\left(\zeta_{5}\right) \Gamma\left(\zeta_{6}\right) \Gamma\left(\zeta_{7}\right) \Gamma\left(\zeta_{8}\right)},
$$


where $\zeta_{1}=\zeta=-i\langle v, \alpha\rangle / 2\langle\alpha, \alpha\rangle, \zeta_{2}=\zeta+\frac{1}{2}, \zeta_{3}=\zeta+\frac{3}{2} j-\frac{3}{4} m-\frac{3}{4} n, \zeta_{4}=\zeta-\frac{3}{2} j+$ $\frac{3}{4} m+\frac{3}{4} n, \zeta_{5}=\zeta+\frac{1}{2} j-\frac{3}{4} m-\frac{3}{4} n, \zeta_{6}=\zeta+\frac{1}{2} j+\frac{1}{4} m-\frac{3}{4} n+1, \zeta_{7}=\zeta-\frac{1}{2} j-\frac{1}{4} m+\frac{3}{4} n$, and $\zeta_{8}=\zeta-\frac{1}{2} j+\frac{3}{4} m+\frac{3}{4} n+1$.

Detailed proofs of these results and some more examples will appear in a paper in preparation.

\section{REFERENCES}

1. M. V. Fedorjuk, The stationary phase method and pseudo-differential operators, Uspehi Mat. Nauk 26 (1971), no. 1 (157), 67-112=Russian Math. Surveys 26 (1971), no. $1,65-111$.

2. Harish-Chandra, Harmonic analysis on semisimple Lie groups, Bull. Amer. Math. Soc. 76 (1970), 529-551. MR 41 \#1933.

3. - On the theory of the Eisenstein integral, Lecture Notes in Math., vol. 266, Springer-Verlag, Berlin, 1972, pp. 123-149.

4. N. Wallach, Harmonic analysis on homogeneous spaces, Marcel Dekker, New York, 1973.

5. - On Harish-Chandra's generalized C-functions, Amer. J. Math. (to appear). 6. G. Warner, Harmonic analysis on semi-simple Lie groups. I, II, Die Grundlehren der math. Wissenschaften, Bände 188, 189, Springer-Verlag, Berlin and New York, 1972.

Department of Mathematics, Johns Hopkins University, Baltimore, MaryLAND 21218 